\title{
On the Performance of Cooperative OFDM-NOMA System with Index Modulation
}

\author{
Xuan Chen, Miaowen Wen, Senior Member, IEEE, and Shuping Dang, Member, IEEE
}

\begin{abstract}
In this letter, we propose a novel three-node cooperative non-orthogonal multiple access (C-NOMA) system based on orthogonal frequency division multiplexing with index modulation (OFDM-IM), which is termed CIM-OFDM-NOMA. In the proposed scheme, the messages for two users are conveyed by two different information-bearing units of OFDM-IM, i.e., $M$-ary signal constellations and the indices of the activated subcarriers, respectively. Furthermore, we consider two different detectors for the cell-edge user, i.e., the maximum likelihood (ML) detector and the low-complexity greedy detector. Based on the ML detector, asymptotically tight bounds on the bit error rate of two users are derived in closed-form. Finally, simulation results verify the theoretical analysis and show that the proposed scheme outperforms the conventional C-NOMA and non-cooperative NOMA with OFDM-IM (IM-NOMA) significantly.
\end{abstract}

Index Terms-Bit error rate, orthogonal frequency division multiplexing, index modulation, non-orthogonal multiple access, cooperative relaying.

\section{INTRODUCTION}

A S a multiple access scheme, non-orthogonal multiple access (NOMA) exhibits a range of advantages over other orthogonal multiple access (OMA) schemes [1], [2]. The key feature of NOMA is to serve multiple users simultaneously by implementing superposition coding (SC) at the transmitter and successive interference cancellation (SIC) at the receiver. However, due to a long transmission distance, the cell-edge users usually suffer from poor communication services. To effectively alleviate this defect, the paradigm of cooperative NOMA (C-NOMA) is proposed [3]. The rationale behind CNOMA is that the cell-center user can act as a relay to forward the information of the cell-edge user, which has been decoded in the SIC process. Recently, the C-NOMA scheme also has been widely studied in terms of capacity, receiver design, and error performance. Nevertheless, C-NOMA is always susceptible to the inter-user interference (IUI) arising from the multiplexing in the power domain. In [4] and [5], the spatial modulation (SM), which is a representative index modulation (IM) scheme, is introduced into C-NOMA systems, forming the SM-aided C-NOMA (CSM-NOMA) scheme. In contrast

Manuscript received February 24, 2020; revised April 2, 2020 and April 14, 2020; accepted April 18, 2020. Date of publication XXXX XX, 2020; date of current version XXXX XX, 2020. This work was supported in part by the Natural Science Foundation of Guangdong Province under Grant 2018B030306005, in part by the Pearl River Nova Program of Guangzhou under Grant 201806010171, in part by the National Natural Science Foundation of China under Grant 61871190, and in part by the State Key Laboratory of Integrated Service Networks of Xidian University under Grant ISN20-16. (Corresponding author: Miaowen wen.)

Xuan Chen and Miaowen Wen are with the School of Electronic and Information Engineering, South China University of Technology, Guangzhou 510640, China, and also with the State Key Laboratory of Integrated Service Networks, Xidian University, Xi' an 710071, China (e-mail: eechenxuan@mail.scut.edu.cn; eemwwen@scut.edu.cn).

Shuping Dang is with Computer, Electrical and Mathematical Sciences, and Engineering Division, King Abdullah University of Science and Technology, Thuwal 23955-6900, Saudi Arabia (e-mail: shuping.dang@kaust.edu.sa).

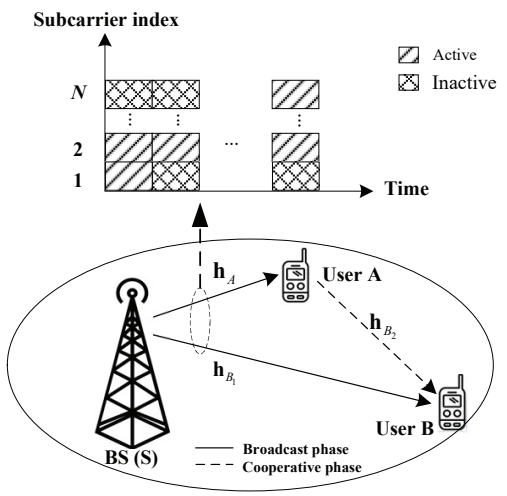

Fig. 1. Schematic diagram of CIM-OFDM-NOMA.

with C-NOMA, CSM-NOMA can avoid the complex SIC decoding procedure and the IUI from the spatial domain. Specifically, the CSM-NOMA systems are realized by exploiting the spatial domain and the two-dimensional signal domain to deliver the information of two users, respectively.

Against this background, in this letter, we propose to eliminate the drawback of C-NOMA from the frequency domain. Similar to SM, the concept of IM has been applied in the frequency domain with orthogonal frequency division multiplexing (OFDM), thus generating a new variant of OFDM termed OFDM-IM [6], [7]. Particularly, OFDM-IM intends to employ the indices of active subcarriers, which carry the $M$-ary modulated constellation symbols, to convey extra information bits (a.k.a. IM bits). Moreover, the transmission of IM bits not only consumes no power or bandwidth, but also has a highly stronger protection mechanism than that of conventional modulation bits [8]. Therefore, we incorporate OFDM-IM into C-NOMA systems, resulting in a new system called CIM-OFDM-NOMA. We illustrate an example with one base station (BS) and two users, i.e., the cell-center and cell-edge users. In CIM-OFDM-NOMA, two different information-bearing units of OFDM-IM, i.e., $M$-ary signal constellations and the indices of the activated subcarriers, are used to convey the messages for two users, respectively. Hence, the information of users will no longer overlap in the power domain, avoiding the IUI, SC, and SIC. The bit error rate (BER) of CIM-OFDM-NOMA is theoretically analyzed, and the corresponding BER bounds are also derived in closed-form. Monte Carlo simulations are carried out to verify the theoretical analysis and compare the performance of CIM-OFDM-NOMA, coded/uncoded C-NOMA, and the noncooperative NOMA with OFDM-IM (IM-NOMA).

\section{SySTEM MODEL}

Consider a downlink CIM-OFDM-NOMA scenario with one BS (S) and two users (the cell-center user A and the celledge user B), as depicted in Fig. 1. We assume that all nodes 
are configured with a single antenna and operate with a halfduplex fashion. The channel coefficients of links $\mathrm{S} \rightarrow \mathrm{A}, \mathrm{S} \rightarrow \mathrm{B}$, and $\mathrm{A} \rightarrow \mathrm{B}$ are represented as $\mathbf{h}_{A}, \mathbf{h}_{B_{1}}$, and $\mathbf{h}_{B_{2}}$, respectively. The entries in $\mathbf{h}_{A}, \mathbf{h}_{B_{1}}$, and $\mathbf{h}_{B_{2}}$ are modeled as independent and identically distributed (i.i.d.) complex Gaussian random variables (r.v.) with distributions $\mathcal{C N}\left(0, \lambda_{S A}\right), \mathcal{C N}\left(0, \lambda_{S B}\right)$, and $\mathcal{C N}\left(0, \lambda_{A B}\right)$, respectively. According to the fact described in [9] that the variance of the channel gain varies inversely with the communication distance, we thereby have $\lambda_{S A}>\lambda_{S B}$. The complete communication protocol consisting of the broadcast phase and the cooperative phase is detailed in the sequel.

\section{A. Broadcast Phase}

During the broadcast phase, the BS first transmits the information of users processed by OFDM-IM on the same frequency and time slot, in which a total of $m$ information bits are conveyed to users $\mathrm{A}$ and $\mathrm{B}$ via an $N \times 1$ OFDM block. Then, this OFDM block is divided into $g$ subblocks, each of which contains $p=m / g$ bits and independently carries out the IM operation within $n=N / g$ subcarriers. Considering that the IM process for each subblock is independent and identical, let us take the $\beta$-th subblock as an example to conduct the follow-up analysis, where $\beta \in\{1, \ldots, g\}$. For clarity, in the $\beta$-th subblock, we define $p=p_{1}+p_{2}$, where $p_{1}$ bits are used to determine $k$ active subcarriers out of $n$ available ones, i.e., $p_{1}=\left\lfloor\log _{2}(n, k)\right\rfloor$; while $p_{2}$ bits are mapped onto conventional signal constellations ( $M$-phase shifting keying (PSK)/quadrature amplitude modulation (QAM)), which are carried by $k$ selected subcarriers, i.e., $p_{2}=k \log _{2}(M)$. Generally, to provide proper communication services for all users, we tend to use $p_{1}$ and $p_{2}$ to serve users $\mathrm{B}$ and $\mathrm{A}$, respectively. Hence, the overall spectral efficiency (SE) of the proposed network is given by $\frac{1}{n}\left(p_{1}+p_{2}\right)$ bits per channel use (bpcu).

For simplicity, we assume that the interleaved subcarrier grouping is used to form $g$ subblocks, so that the subcarriers within each subcarrier subblock are independently faded [10]. Hence, we can write the indices of the selected subcarriers as

$$
I^{(\beta)}=\left\{i^{(\beta)}(1), \ldots, i^{(\beta)}(k)\right\},
$$

where $i^{(\beta)}(\gamma) \in\{\beta, \ldots, \beta+(\gamma-1) g, \ldots, \beta+(n-1) g\}$ for $\gamma=1, \ldots, k$. The PSK/QAM symbol vector carrying $p_{2}$ bits is represented by

$$
\mathbf{s}^{(\beta)}=\left[s^{(\beta)}(1), s^{(\beta)}(2), \ldots, s^{(\beta)}(k)\right]^{T},
$$

where $s^{(\beta)}(\gamma) \in \mathcal{X}$ denotes the modulated symbol of user A, and $\mathcal{X}$ represents the complex PSK/QAM constellation set of cardinality $M$. Afterwards, the transmitted vector for the $\beta$-th subblock can be written as

$\mathbf{x}^{(\beta)}=[x(\beta), \ldots, x(\beta+(\gamma-1) g), \ldots, x(\beta+(n-1) g)]^{T}$,

where $x^{(\beta)}(\lambda) \in\{0, \mathcal{X}\}$ with $\lambda=\{1, \ldots, n\}$. All OFDM subblocks are concatenated to generate a new OFDM block. The remaining procedures are the same as those of the classical OFDM, including the inverse fast Fourier transform (IFFT), cyclic prefix (CP) appending, and parallel-to-series conversion.

At the receiving side, after removing the $\mathrm{CP}$ and performing the fast Fourier transform (FFT), the frequency-domain received signals on the $\beta$-th subblock at users $\mathrm{A}$ and $\mathrm{B}$ can be expressed as

$$
\mathbf{y}_{A}^{(\beta)}=\mathbf{X}^{(\beta)} \mathbf{h}_{A}^{(\beta)}+\mathbf{w}_{A}^{(\beta)},
$$

and

$$
\mathbf{y}_{B_{1}}^{(\beta)}=\mathbf{X}^{(\beta)} \mathbf{h}_{B_{1}}^{(\beta)}+\mathbf{w}_{B_{1}}^{(\beta)}
$$

respectively, where $\mathbf{X}^{(\beta)}=\operatorname{diag}\left(\mathbf{x}^{(\beta)}\right) ; \mathbf{w}_{A}^{(\beta)}$ and $\mathbf{w}_{B_{1}}^{(\beta)}$ are the additive white Gaussian noise (AWGN) vectors for links $\mathrm{S} \rightarrow \mathrm{A}$ and $\mathrm{S} \rightarrow \mathrm{B}$, respectively, both following the distribution $\mathcal{C N}\left(0, N_{0}\right)$. Finally, user $\mathrm{B}$ retains the received signal $\mathbf{y}_{B_{1}}^{(\beta)}$ for demodulation in the next phase, while user A employs the maximum likelihood (ML) detector to recover the transmitted signals. Hence, the detection process can be expressed as

$$
\left(\tilde{I}^{(\beta)}, \hat{\mathbf{s}}^{(\beta)}\right)=\underset{I^{(\beta)}, \mathbf{s}^{(\beta)}}{\arg \min }\left\|\mathbf{y}_{A}^{(\beta)}-\mathbf{X}^{(\beta)} \mathbf{h}_{A}^{(\beta)}\right\|^{2} .
$$

Here, note that user A further recovers its own information bits from $\hat{\mathbf{S}}^{(\beta)}$ and simultaneously preserves $\tilde{I}^{(\beta)}$ for cooperation.

\section{B. Cooperative Phase}

From (6), it is clear that user A can utilize $\tilde{I}^{(\beta)}$ to regenerate a new OFDM-IM signal $\tilde{\mathbf{x}}_{B_{2}}^{(\beta)}$ through the same procedures as (3). Unlike (2), the modulated symbol in $\tilde{\mathbf{x}}_{B_{2}}^{(\beta)}$ is set to one in all active subcarriers. Next, $\tilde{\mathbf{x}}_{B_{2}}^{(\beta)}$ is forwarded to user B with the full transmit power. The received signal at user B is similarly given by

$$
\mathbf{y}_{B_{2}}^{(\beta)}=\tilde{\mathbf{X}}_{B_{2}}^{(\beta)} \mathbf{h}_{B_{2}}^{(\beta)}+\mathbf{w}_{B_{2}}^{(\beta)},
$$

where $\tilde{\mathbf{X}}_{B_{2}}^{(\beta)}=\operatorname{diag}\left(\tilde{\mathbf{x}}_{B_{2}}^{(\beta)}\right)$, and $\mathbf{w}_{B_{2}}^{(\beta)}$ is the AWGN vector for the link $\mathrm{A} \rightarrow \mathrm{B}$, distributed as $\mathcal{C} \mathcal{N}\left(0, N_{0}\right)$. Then, user B can make a decision based on the two observations, namely $\mathbf{y}_{B_{1}}^{(\beta)}$ and $\mathbf{y}_{B_{2}}^{(\beta)}$. Attempting to reduce the detection complexity, we propose two different detectors as follows.

1) ML Detector: According to the ML criterion, user B can extract its own information by

$$
\begin{array}{r}
\hat{I}^{(\beta)}=\underset{I^{(\beta)}}{\arg \min }\left\{\min _{\mathbf{s}^{(\beta)}}\left\|\mathbf{y}_{B_{1}}^{(\beta)}-\mathbf{X}^{(\beta)} \mathbf{h}_{B_{1}}^{(\beta)}\right\|^{2}\right. \\
\left.+\left\|\mathbf{y}_{B_{2}}^{(\beta)}-\tilde{\mathbf{X}}_{B_{2}}^{(\beta)} \mathbf{h}_{B_{2}}^{(\beta)}\right\|^{2}\right\},
\end{array}
$$

where $\hat{I}^{(\beta)}$ is the estimate of $I^{(\beta)}$. Obviously, the computational complexity of the optimal ML detector in terms of the number of complex multiplications is $\mathcal{O}\left(2^{\left\lfloor\log _{2}(n, k)\right\rfloor}\left(1+M^{k}\right)\right)$.

2) Greedy Detector: Considering that there are two different received signals at user B, the greedy (GD) detector estimates $k$ active subcarriers out of $n$ available ones by determining $k$ maximum combined energies, i.e.,

$$
\hat{I}^{(\beta)}=\underset{I^{(\beta)}}{\arg \max }\left\{\mathcal{Y}_{1}+\mathcal{Y}_{2}\right\},
$$

where we have

$$
\begin{aligned}
& \mathcal{Y}_{1}=\left|y_{B_{1}}^{(\beta)}\left(i^{(\beta)}(1)\right)\right|^{2}+\cdots\left|y_{B_{1}}^{(\beta)}\left(i^{(\beta)}(k)\right)\right|^{2} \\
& \mathcal{Y}_{2}=\left|y_{B_{2}}^{(\beta)}\left(i^{(\beta)}(1)\right)\right|^{2}+\cdots\left|y_{B_{2}}^{(\beta)}\left(i^{(\beta)}(k)\right)\right|^{2}
\end{aligned}
$$


Here, notice that CIM-OFDM-NOMA may not make use of all $C(n, k)$ subcarrier activation patterns, but only $2^{\left\lfloor\log _{2}(n, k)\right\rfloor}$ of them are exploited. Therefore, the GD detector makes a further decision as follows. We first define $\mathcal{I}$ as all possible subcarrier activation patterns. If $\hat{I}^{(\beta)} \in \mathcal{I}$, the estimate of $I^{(\beta)}$ can be directly determined by $\hat{I}^{(\beta)}$. Otherwise, if $\hat{I}^{(\beta)} \notin \mathcal{I}$, the pattern $\check{I}$ with the minimum distance from $\hat{I}^{(\beta)}$ is selected as the estimate of $I^{(\beta)}$, i.e., $\hat{I}^{(\beta)}=\breve{I}$. From (9), it is clear that the computational complexity is $\mathcal{O}\left(2^{\left\lfloor\log _{2}(n, k)\right\rfloor}\right)$, which is much lower than that of the ML detector.

\section{Performance Analysis}

In this section, we derive upper bounds on the BER of CIMOFDM-NOMA assuming perfect channel estimation and the ML detection. Meanwhile, the superscript $(\beta)$ in all notations is omitted for clarity in the subsequent analysis.

\section{A. BER of User A}

From (6), user A can recover its own signal $s$ from the received symbol $\mathbf{y}_{A}$ via the ML detector. Obviously, the bit errors of user A can be divided into two cases, depending on whether the index of the activated subcarrier is correctly detected. Therefore, we first study the index error probability (IEP) to characterize the bit error probabilities (BEPs) for these two cases, and then obtain an overall BER upper bound. For brevity, we define $P(I \rightarrow \hat{I})$ as the probability of detecting $I$ as $\hat{I}$, which can be computed by

$$
P(I \rightarrow \hat{I})=\frac{1}{2^{p_{2}}} \sum_{\mathbf{s}, \hat{\mathbf{s}}} \operatorname{Pr}(\mathbf{X} \rightarrow \hat{\mathbf{X}} \mid \mathbf{s}, \hat{\mathbf{s}}, I, \hat{I}),
$$

where $\operatorname{Pr}(\mathbf{X} \rightarrow \hat{\mathbf{X}} \mid \mathbf{s}, \hat{\mathbf{s}}, I, \hat{I})$ is the probability of detecting $\mathbf{X}$ as $\hat{\mathbf{X}}$ conditioned on $\mathbf{s}, \hat{\mathbf{s}}, I$, and $\hat{I}$, and it can be obtained via averaging $\operatorname{Pr}\left(\mathbf{X} \rightarrow \hat{\mathbf{X}} \mid \mathbf{s}, \hat{\mathbf{s}}, I, \hat{I}, \mathbf{h}_{A}\right)$ over $\mathbf{h}_{A}$. Here, $\operatorname{Pr}\left(\mathbf{X} \rightarrow \hat{\mathbf{X}} \mid \mathbf{s}, \hat{\mathbf{s}}, I, \hat{I}, \mathbf{h}_{A}\right)$ can be formulated as

$$
\operatorname{Pr}\left(\mathbf{X} \rightarrow \hat{\mathbf{X}} \mid \mathbf{s}, \hat{\mathbf{s}}, I, \hat{I}, \mathbf{h}_{A}\right)=Q\left(\sqrt{\frac{\left\|(\mathbf{X}-\hat{\mathbf{X}}) \mathbf{h}_{A}\right\|^{2}}{2 N_{0}}}\right) .
$$

Here, we can approximate (11) quite well by employing

$$
Q(x) \approx \frac{1}{12} e^{-x^{2} / 2}+\frac{1}{4} e^{-2 x^{2} / 3} .
$$

Furthermore, by applying the method proposed in [7], $\operatorname{Pr}(\mathbf{X} \rightarrow \hat{\mathbf{X}} \mid \mathbf{s}, \hat{\mathbf{s}}, I, \hat{I})$ can be written as

$$
\begin{aligned}
& \operatorname{Pr}(\mathbf{X} \rightarrow \hat{\mathbf{X}} \mid \mathbf{s}, \hat{\mathbf{s}}, I, \hat{I}) \\
& \approx \frac{1 / 12}{\operatorname{det}\left(\mathbf{I}_{n}+q_{1} \overline{\mathbf{K}}_{n} \mathbf{A}\right)}+\frac{1 / 4}{\operatorname{det}\left(\mathbf{I}_{n}+q_{2} \overline{\mathbf{K}}_{n} \mathbf{A}\right)},
\end{aligned}
$$

where $q_{1}=1 /\left(4 N_{0}\right), q_{2}=1 /\left(3 N_{0}\right), \mathbf{A}=(\mathbf{X}-\hat{\mathbf{X}})^{H}(\mathbf{X}-$ $\hat{\mathbf{X}})$, and $\overline{\mathbf{K}}_{n}=E\left\{\mathbf{h}_{A} \mathbf{h}_{A}^{H}\right\}=\mathbf{I}_{n}$ with $\mathbf{I}_{n}$ denoting the $n \times n$ identity matrix. Substituting (13) into (10) yields the final expression of $P(I \rightarrow \hat{I})$. Having obtained $P(I \rightarrow \hat{I})$, the overall BER of user A can be calculated using the union bound technique, given by [11]

$$
P_{A} \leq \frac{1}{2^{p_{1}}} \sum_{I, \hat{I}} P(I \rightarrow \hat{I})\left[P_{A b} D(I, \hat{I})+\frac{1}{2} \bar{D}(I, \hat{I})\right],
$$

where $D(I, \hat{I})$ and $\bar{D}(I, \hat{I})$ denote the numbers of the activated subcarriers detected correctly or incorrectly, respectively. $P_{A b}$ is the average BEP of $M$-PSK/QAM. Specifically, for $M$-PSK over a Rayleigh fading channel, $P_{A b}$ can be determined as [12]

$$
\begin{aligned}
P_{A b}(M) & =\left(\frac{M-1}{M}\right)\left\{1-\sqrt{\frac{g_{\mathrm{PSK}} \rho_{b}}{1+g_{\mathrm{PSK}} \rho_{b}}}\left(\frac{M}{(M-1) \pi}\right)\right. \\
& \left.\times\left[\frac{\pi}{2}+\tan ^{-1}\left(\sqrt{\frac{g_{\mathrm{PSK}} \rho_{b}}{1+g_{\mathrm{PSK}} \rho_{b}}} \cot \frac{\pi}{M}\right)\right]\right\},
\end{aligned}
$$

where $\rho_{b}=\lambda_{A B} \rho / \log _{2}(M), g_{\mathrm{PSK}} \triangleq \sin ^{2} \pi / M$, and $\rho$ denotes the signal-to-noise ratio (SNR). Correspondingly, the expression of $P_{A b}$ for $M$-QAM can also be found from [12]. Further, we can observe from (14) that the diversity order of user $\mathrm{A}$ is determined by $P_{A b}(M)$, which yields unity.

\section{B. BER of User B}

In this subsection, we first recall that bit error events for user B can mainly be classified into two categories: a) user B detects $I$ incorrectly, while $I$ has been decoded well at user A; b) user A detects $I$ incorrectly. Therefore, we can obtain the BER expression of user B by

$$
P_{B} \approx \frac{1}{p_{1}}\left(1-P_{I A}\right) P_{I B}+\frac{1}{2} P_{I A},
$$

where $P_{I A}$ represents the IEP at user $\mathrm{A}$, and $P_{I B}$ is the IEP at user B when $I$ is detected correctly at user A. Additionally, it is conservatively assumed that the BEP of user B is $1 / 2$ if $I$ has already been erroneously detected at user A. As for $P_{I A}$, according to (10), we have

$$
P_{I A} \leq \frac{1}{2^{p_{1}}} \sum_{I, \hat{I}} P(I \rightarrow \hat{I}) .
$$

Furthermore, let us utilize (8) to derive the conditional pair error probability (PEP), expressed as

$$
\begin{aligned}
& \operatorname{Pr}\left(\mathbf{X} \rightarrow \hat{\mathbf{X}} \mid \mathbf{h}_{B_{1}}, \mathbf{h}_{B_{2}}, \mathbf{s}, \hat{\mathbf{s}}\right) \\
& =\operatorname{Pr}\left(\left\|\mathbf{y}_{B_{1}}-\mathbf{X h}_{B_{1}}\right\|^{2}+\left\|\mathbf{y}_{B_{2}}-\mathbf{X}_{B_{2}} \mathbf{h}_{B_{2}}\right\|^{2}\right. \\
& \left.\quad>\left\|\mathbf{y}_{B_{1}}-\hat{\mathbf{X}} \mathbf{h}_{B_{1}}\right\|^{2}+\left\|\mathbf{y}_{B_{2}}-\hat{\mathbf{X}}_{B_{2}} \mathbf{h}_{B_{2}}\right\|^{2}\right) \\
& =Q\left(\sqrt{\frac{1}{2 N_{0}} \sum_{\gamma=1}^{n} T(\gamma)}\right) .
\end{aligned}
$$

Here, notice that $\mathbf{X}_{B_{2}}$ is constructed by the correct estimate of $I$ at user A. Therefore, using the approximation of $Q$-function (13), the unconditional PEP can be written as

$$
\begin{aligned}
\operatorname{Pr}(\mathbf{X} \rightarrow \hat{\mathbf{X}} \mid \mathbf{s}, \hat{\mathbf{s}}) & \approx \frac{1}{12} \prod_{\gamma=1}^{n} E_{\mathbf{h}_{B_{1}}, \mathbf{h}_{B_{2}}}\left\{\exp \left(-q_{1} T(\gamma)\right)\right\} \\
& +\frac{1}{4} \prod_{\gamma=1}^{n} E_{\mathbf{h}_{B_{1}}, \mathbf{h}_{B_{2}}}\left\{\exp \left(-q_{2} T(\gamma)\right)\right\},
\end{aligned}
$$

where $q_{1}$ and $q_{2}$ follow the same definitions as (13). For (18) and (19), we have

$$
\begin{aligned}
T(\gamma)= & \left|(x(\gamma)-\hat{x}(\gamma)) h_{B_{1}}(\gamma)\right|^{2} \\
& +\left|\left(x_{B_{2}}(\gamma)-\hat{x}_{B_{2}}(\gamma)\right) h_{B_{2}}(\gamma)\right|^{2} .
\end{aligned}
$$


Obviously, it can be found that $T(\gamma)$ is the sum of two independent central chi-square random variables. Hence, the moment generating function (MGF) of $T(\gamma)$ is given by [13]

$$
\mathcal{M}(t)=\frac{1}{\left(1-2 t \sigma_{1}^{2}\right)\left(1-2 t \sigma_{2}^{2}\right)},
$$

where

$$
\begin{aligned}
\sigma_{1}^{2} & =\lambda_{S B}|x(\gamma)-\hat{x}(\gamma)|^{2} \\
\sigma_{2}^{2} & =\lambda_{A B}\left|x_{B_{2}}(\gamma)-\hat{x}_{B_{2}}(\gamma)\right|^{2} .
\end{aligned}
$$

Resorting to the MGF approach, we can rewrite (19) as

$$
\operatorname{Pr}(\mathbf{X} \rightarrow \hat{\mathbf{X}} \mid \mathbf{s}, \hat{\mathbf{s}}) \approx \frac{1}{12} \prod_{\gamma=1}^{n} \mathcal{M}\left(-q_{1}\right)+\frac{1}{4} \prod_{\gamma=1}^{n} \mathcal{M}\left(-q_{2}\right)
$$

Therefore, the IEP of user B with the ML detector can be written as

$$
P_{I B} \leq \frac{1}{2^{p}} \sum_{I, \hat{I}} \sum_{\mathbf{s}, \hat{\mathbf{s}}} \operatorname{Pr}(\mathbf{X} \rightarrow \hat{\mathbf{X}} \mid \mathbf{s}, \hat{\mathbf{s}}) .
$$

Finally, the final BER expression for user B can be obtained by substituting (17) and (23) into (16). It is clear from (16) that the diversity order of user $\mathrm{B}$ is the smaller one between the two diversity orders captured by $P_{I A}$ and $P_{I B}$. According to [7], $P_{I A}$ exhibits a diversity order of $\operatorname{rank}(\mathbf{A})$. Similar to $P_{I A}$, the diversity order of $P_{I B}$ in (23) is $\operatorname{rank}(\mathbf{A})+\operatorname{rank}(\mathbf{B})$, where $\mathbf{B}=\left(\mathbf{X}_{B_{2}}-\hat{\mathbf{X}}_{B_{2}}\right)^{H}\left(\mathbf{X}_{B_{2}}-\hat{\mathbf{X}}_{B_{2}}\right)$. Therefore, user B can achieve a diversity order of $\operatorname{rank}(\mathbf{A})$.

\section{Simulation Results and Discussion}

In this section, we employ Monte Carlo simulations to demonstrate the uncoded BER performance of the proposed scheme, and include coded/uncoded C-NOMA investigated in [4] and the IM-NOMA scheme as benchmarks to measure the system performance, where a convolutional code with half-rate is employed for coded C-NOMA and its generator function is $\mathrm{g}_{0}=(111)$ and $\mathrm{g}_{1}=(101)$. Unlike the proposed scheme, there is no cooperation between users in IM-NOMA. For fair comparison, in all simulations, we assume that each subcarrier experiences independent Rayleigh fading, whose channel coefficient is perfectly known to users $\mathrm{A}$ and $\mathrm{B}$. Meanwhile, we set $\lambda_{S B}=1, \lambda_{S A}=6$, and $\lambda_{A B}=2$. For brevity, we refer to "CIM-OFDM-NOMA/IM-NOMA ( $M$ PSK, $n, k)$ " as the CIM-OFDM-NOMA/IM-NOMA scheme, in which only $k$ subcarriers out of $n$ available ones per group are activated to convey the $M$-PSK symbols at the BS, and "C-NOMA ( $M_{A}$-PSK, $M_{B}$-PSK)" as the C-NOMA scheme, in which the BS maps the information bits for users $\mathrm{A}$ and $\mathrm{B}$ into $M_{A}$-PSK and $M_{B}$-PSK symbols, respectively.

\section{A. Validation of Theoretical Results}

In this subsection, we compare the theoretical results with their simulation counterparts to verify the derived BERs.

Fig. 2 illustrates the theoretical BER of CIM-OFDMNOMA with the ML detector, as well as the corresponding simulation results. It can be clearly figured out from Fig. 2 that the derived BER upper bounds of user A accurately

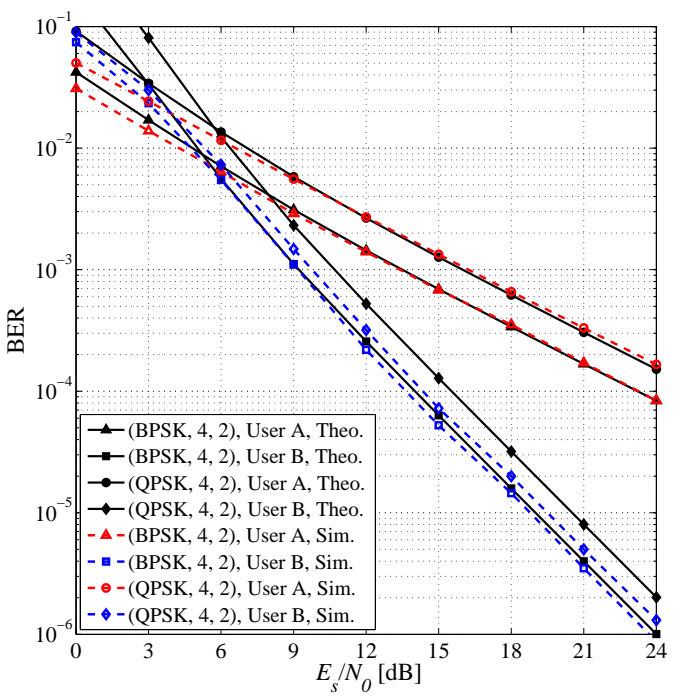

Fig. 2. BER versus $E_{s} / N_{0}$ performance of the CIM-OFDM-NOMA scheme: numerical verification, where $E_{s}$ is the average transmitted energy per symbol.

match their simulation counterparts in the SNR range of interest with different values of $M$. On the other hand, we can observe that the BER upper bounds of user B with the ML detector gradually become looser as $M$ goes large, although the theoretical results agree with the simulation counterparts perfectly in the high SNR region when $M=2$. This can be understood by the fact that $P_{I B}$ from (23) is excessively enlarged with the increase of $M$.

\section{B. Performance of CIM-OFDM-NOMA with Different Settings}

In this subsection, the achievable BER versus $E_{b} / N_{0}$ performance of CIM-OFDM-NOMA with different settings is investigated, which are further compared with those of coded/uncoded C-NOMA and IM-NOMA. Notice that $E_{b}$ is the average transmitted energy per bit. Considering that user A of IM-NOMA performs the same process as that of CIM-OFDMNOMA, the BER curves of user A for IM-NOMA are not given. Moreover, the values of $\alpha$ are 0.17/0.22 and 0.19/0.29 for coded/uncoded C-NOMA in Figs. 3 and 4, respectively. ${ }^{1}$

In Fig. 3, we compare the BER performance among CIMOFDM-NOMA, coded/uncoded C-NOMA, and IM-NOMA, whose SEs are $2.25,2$, and $2.25 \mathrm{bpcu}$, respectively. It is clear from Fig. 3 that both users of the proposed scheme outperform those of uncoded C-NOMA in terms of the SE and BER performance, when the ML detector is employed. Specifically, SNR gains of up to $6 \mathrm{~dB}$ and $9 \mathrm{~dB}$ for users $\mathrm{A}$ and $\mathrm{B}$ are attained at BER $=10^{-3}$. These advantages can be accounted for by two reasons: one is that the interference among users has been greatly alleviated by assigning different information-bearing units to different users; the other is the nearly transparent delivery of IM bits without power consumption. Alternatively, thanks to the diversity gain from OFDMIM, the SNR gain for user B becomes increasingly large with

\footnotetext{
${ }^{1}$ The optimal power allocation factor $\alpha$ has been obtained by the exhaustive computer search following [4].
} 


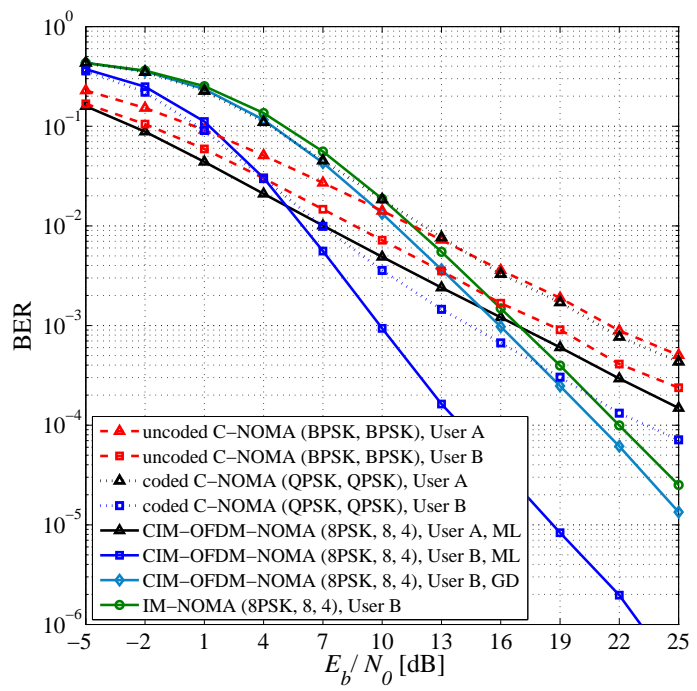

Fig. 3. BER comparison among "CIM-OFDM-NOMA (8PSK, 8, 4)", "coded C-NOMA (QPSK, QPSK)", "uncoded C-NOMA (BPSK, BPSK)", and "IMNOMA (8PSK, 8, 4)".

a decreasing BER. Similar to uncoded C-NOMA, we can observe that the proposed scheme also performs better than coded C-NOMA, although the latter attains an extra channel coding gain. Moreover, comparing the proposed scheme with IM-NOMA, we can find the cooperative link brings about $7 \mathrm{~dB}$ SNR gain at the cost of an additional time slot. As for the GD detector, it is clear from Fig. 3 that compared with the ML detector, the performance loss of the GD detector is about $6 \mathrm{~dB}$. However, the performance distortion arising from the reduced complexity is inevitable. Fortunately, the performance of CIM-OFDM-NOMA with the GD detector is still superior to that of uncoded C-NOMA and IM-NOMA at user B.

Fig. 4 gives the comparison results when the SE of CIMOFDM-NONA/IM-NOMA increases from 2.25 bpcu to 3.125 bpcu. Correspondingly, C-NOMA achieves an SE of 3 bpcu. It can be observed from Fig. 4 that the proposed scheme still retain the superiority. Specifically, SNR gains of $7 \mathrm{~dB}, 8 \mathrm{~dB}$, and $14 \mathrm{~dB}$ for user $\mathrm{B}$ at BER $=10^{-4}$ are attained against IMNOMA and coded/uncoded C-NOMA. As for user A, coded C-NOMA exhibits the worst performance in all simulations, which is mainly due to the higher modulation order and the serious error propagation of SIC; while constrained on the size of signal constellation and different transmit power allocation strategies, all other schemes have similar performance.

\section{CONCLUSION}

In this letter, we have proposed the CIM-OFDM-NOMA scheme, in which the techniques of C-NOMA and IM are integrated based on the OFDM framework to mitigate the IUI and reduce the implementation complexity. The core idea behind the proposed scheme is to use two different information-bearing units of OFDM-IM to serve the cellcenter and cell-edge users, respectively. Moreover, a lowcomplexity GD detector has been designed for the celledge user. Finally, we have proven that the proposed scheme

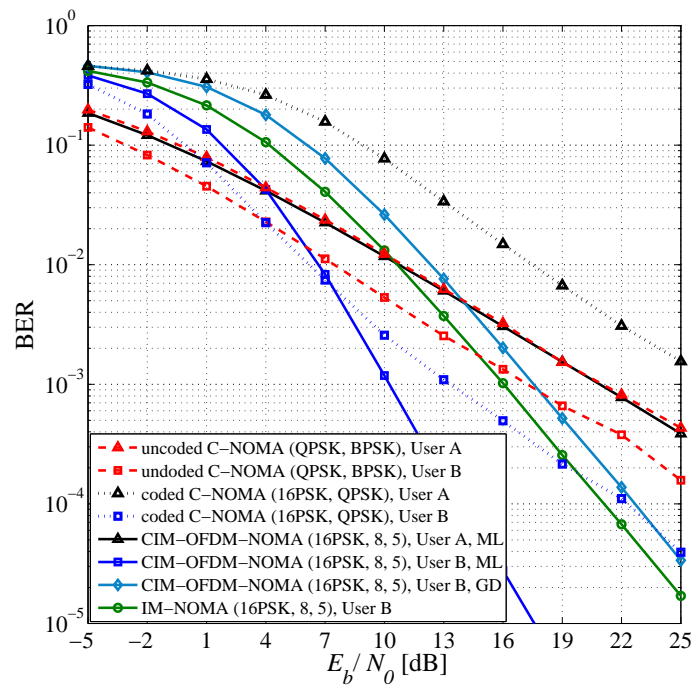

Fig. 4. BER comparison among "CIM-OFDM-NOMA (16PSK, 8, 5)", "coded C-NOMA (16PSK, QPSK)", "uncoded C-NOMA (QPSK, BPSK)", and "IM-NOMA (16PSK, 8, 5)".

significantly outperforms C-NOMA and IM-NOMA through theoretical analysis and numerical simulations.

\section{REFERENCES}

[1] L. Dai, B. Wang, Y. Yuan, S. Han, C. I, and Z. Wang, "Non-orthogonal multiple access for 5G: solutions, challenges, opportunities, and future research trends," IEEE Commun. Mag., vol. 53, no. 9, pp. 74-81, Sep. 2015.

[2] Z. Wei, L. Yang, D. W. K. Ng, J. Yuan, and L. Hanzo, "On the performance gain of NOMA over OMA in uplink communication systems," IEEE Trans. Commun., vol. 68, no. 1, pp. 536-568, Jan. 2020.

[3] Z. Ding, M. Peng, and H. V. Poor, "Cooperative non-orthogonal multiple access in 5G systems," IEEE Commun. Lett., vol. 19, no. 8, pp. 14621465, Aug. 2015.

[4] Q. Li, M. Wen, E. Basar, H. V. Poor, and F. Chen, "Spatial modulationaided cooperative NOMA: performance analysis and comparative study," IEEE J. Sel. Topics Signal Process., vol. 13, no. 3, pp. 715-728, June 2019.

[5] L. Pan and J. Zheng, "Spatial modulation aided cooperative NOMA: implementation and achievable rate analysis," in Proc. IEEE Int. Conf. Commun. (ICC), Shanghai, China, May 2019, pp. 1-6.

[6] S. Sugiura, T. Ishihara, and M. Nakao, "State-of-the-art design of index modulation in the space, time, and frequency domains: Benefits and fundamental limitations," IEEE Access, vol. 5, pp. 21774-21790, Oct. 2017.

[7] E. Basar, U. Ayglu, E. Panayirci, and H. V. Poor, "Orthogonal frequency division multiplexing with index modulation," IEEE Trans. Signal Process., vol. 61, no. 22, pp. 5536-5549, Nov. 2013.

[8] M. Wen, B. Ye, E. Basar, Q. Li, and F. Ji, "Enhanced orthogonal frequency division multiplexing with index modulation," IEEE Trans. Wireless Commun., vol. 16, no. 7, pp. 4786-4801, July 2017.

[9] Z. Ding, Z. Yang, P. Fan, and H. V. Poor, "On the performance of non-orthogonal multiple access in 5G systems with randomly deployed users," IEEE Signal Process. Lett., vol. 21, no. 12, pp. 1501-1505, Dec. 2014.

[10] M. Wen, X. Cheng, M. Ma, B. Jiao, and H. V. Poor, "On the achievable rate of OFDM with index modulation," IEEE Trans. Signal Process., vol. 64, no. 8, pp. 1919-1932, Apr. 2016.

[11] M. Wen, X. Chen, Q. Li, E. Basar, Y. Wu, and W. Zhang, "Index modulation aided subcarrier mapping for dual-hop OFDM relaying," IEEE Trans. Commun., vol. 67, no. 9, pp. 6012-6024, Sept. 2019.

[12] M. K. Simon and M.-S. Alouini, Digital Communication Over Fading Channels, 2nd ed. New York, NY, USA: Wiley, 2005.

[13] M. K. Simon, Probability Distributions Involving Gaussian Random Variables: A Handbook for Engineers and Scientists. Berlin, Germany: Springer, 2006. 